Reprod. Nutr. Dévelop., 1986, 26 (1 B), 375-376.

\title{
Enzymologie hépatique de biotransformation chez l'agneau. Description et évolution en cours de fasciolose
}

P. GALTIER, G. LARRIEU

Laboratoire de Pharmacologie-Toxicologie, I.N.R.A. 180, chemin de Tournefeuille, 31300 Toulouse.

Summary. Fascioliasis has been produced in the lamb by an oral administration of 150 metacercariae of Fasciola hepatica. The cytochrome P 450 and b5 concentrations and ethoxycoumarin 0-deethylase activity fell by weeks 4 and 8 post-infestation whereas both $\mathrm{NADPH}$ cytochrome $\mathrm{c}$ reductase and aminopyrine $\mathrm{N}$-demethylase were only decreased by week 8.

Le manque d'information concernant l'enzymologie hépatique de biotransformation des médicaments chez les ruminants nous a conduits à déterminer les principales activités caractéristiques des microsomes et du cytosol hépatocytaires chez l'agneau. Ayant précédemment démontré l'incidence de la fasciolose expérimentale sur le métabolisme médicamenteux par le foie de rat (Galtier et al., 1983), nous avons entrepris une étude comparable chez des agneaux infestés par 150 métacercaires de Fasciola hepatica.

Matériel et méthodes. Des agneaux mâles sevrés, issus de croisement Berrichon/Romanov et pesant 16 à $24 \mathrm{~kg}$, ont été répartis en 3 lots de 4 animaux comprenant un lot témoin non infesté et 2 lots infestés par l'administration orale de 150 métacercaires de Fasciola hepatica. Nourris (régime mixte : foin-granulés, 90-10\%) et abreuvés à volonté, les agneaux ont été sacrifiés par groupes de six ( 2 témoins +4 infestés) 4 et 8 semaines après l'infestation. Lors de l'abattage, un échantillon de sang était recueilli puis le foie était rapidement prélevé, essuyé, pesé $\left(0-4^{\circ} \mathrm{C}\right)$ puis découpé en 7 fragments, mis en congélation $\left(-80^{\circ} \mathrm{C}\right)$ en attendant le fractionnement. Les microsomes et le cytosol des hépatocytes étaient préparés par centrifugations successives à $10000 \mathrm{~g}(20 \mathrm{~min})$ puis $105000 \mathrm{~g}(1 \mathrm{~h})$, le culot correspondant aux microsomes étant mis en suspension

TABL. 1. - Evolution des systèmes enzymatiques de biotransformations hépatiques chez l'agneau en cours de fasciolose (moyenne de 4 animaux par lot).

\begin{tabular}{|c|c|c|c|}
\hline \multirow{2}{*}{$\begin{array}{c}\text { Activités } \\
(\text { nmoles } / \mathrm{mg} \text { ou } / \mathrm{mg} \times \mathrm{mn})\end{array}$} & \multirow{2}{*}{ Témoins } & \multicolumn{2}{|c|}{ Semaine après l'infestation } \\
\hline & & & \\
\hline Cvtochrome P 450 & $0,288 \pm 0,023$ & $0,253 \pm 0,023^{*}$ & $0,223 \pm 0,015^{*}$ \\
\hline Cytochrome b5 & $0,188 \pm 0,004$ & $0,168 \pm 0,013^{*}$ & $0,166 \pm 0,029^{*}$ \\
\hline NADPH cyt. c reductase (NCR) & $25,9 \pm 2,0$ & $26,0 \pm 3,2$ & $22,6 \pm 1,5^{*}$ \\
\hline Ethoxycoumarin 0 -deethylase (EDE) & $0,724 \pm 0,091$ & $0,484 \pm 0,199^{*}$ & $0,500 \pm 0,109^{*}$ \\
\hline Aminopyrine $\mathrm{N}$-demethylase (AD) & $0,207 \pm 0,021$ & $0,207 \pm 0,073$ & $0,173 \pm 0,048^{*}$ \\
\hline Aniline hydroxylase $(\mathrm{AH})$ & $0,132 \pm 0,014$ & $0,153 \pm 0,047$ & $0,142 \pm 0,009$ \\
\hline Glucuronyltransférase (GCT) & $2,14 \pm 0,16$ & $2,09 \pm 0,28$ & $2,06 \pm 0,48$ \\
\hline Glutathiotransférase (GTT) & $1,40 \pm 0,30$ & $\pm 0,11$ & $\pm 0,07$ \\
\hline
\end{tabular}

\footnotetext{
* Résultat significativement différent de celui des animaux témoins $(P<0.05)$.
} 
dans du tampon phosphate $\mathrm{Na} / \mathrm{K}(0,1 \mathrm{M} ; \mathrm{pH} 7,4)$. Les activités plasmatiques des glutamyltransférase ( $\gamma \mathrm{GT})$, glutamate déshydrogénase (GLDH) et sorbitol déshydrogénase (SDH) étaient mesurées à l'aide des Biochemica Test Combination correspondants (Boehringer Mannheim). Les concentrations en cytochrome P 450 et b5 et les activités hépatiques de biotransformation rapportées dans le tableau 1 ont été mesurées selon la méthodologie déjà décrite (Galtier et al., 1984).

Résultats et discussion. A l'autopsie, les lésions caractéristiques de la fasciolose étaient observées dès que la quatrième semaine après l'infestation. L'atteinte hépatique était confirmée par l'augmentation significative des $\gamma$ GT (19 et $90 \%$ ), $\mathrm{SDH}(80$ et $182 \%$ ) et $\mathrm{GLDH}(85$ et $541 \%$ ) plasmatiques aux semaines 4 et 8 après l'infestation. Par contre, on n'enregistrait aucune variation notable du poids de foie ou des protéines microsomales et cytosoliques. Les valeurs des différentes activités enzymatiques de biotransformation hépatiques relevées chez les agneaux témoins et parasités sont rapportées dans le tableau 1. Une diminution significative des concentrations microsomales en cytochrome P 450 et b5 $(10,6$ à $22 \%)$ est obtenue après 1 ou 2 mois de parasitisme ; elle est corrélée avec celle, plus marquée, de l'EDE (31-33\%). La NCR et I'AD ne sont inhibées qu'en $8^{e}$ semaine de l'infestation. Les enzymes de conjugaison ne subissaient aucune variation liée au parasitisme alors que l'activité de I'ACT cytosolique se situait à un niveau inférieur au seuil de sensibilité de la méthode utilisée $(0.05 \mathrm{nmole} / \mathrm{mg} \times \mathrm{min})$. En comparaison avec des résultats précédemment obtenus (Galtier et al., 1984), l'agneau possède un équipement microsomal en cytochromes équivalent à celui du rat ; toutefois, l'EDE, la GCT et GTT sont 2 à 7 fois plus actives, le pouvoir hydroxylateur de l'aniline étant, par contre, 8 fois moins efficace. La raison du défaut d'acétylation de l'isoniazide par le cytosol hépatique devra être précisée, soit absence générale de cette enzyme chez les ovins, soit polymorphisme phénotypique comme chez l'Homme ou le lapin (Weber et Glowinski, 1980).

L'incidence de la fasciolose sur les activités enzymatiques de biotransformation hépatique de l'agneau se mesure essentiellement au niveau du cytochrome $P 450$ et des activités qui en dépendent: AD, EDE. Ces conclusions sont en accord avec celles obtenues chez le rat (Galtier et al., 1983). Toutefois la baisse de la NCR démontre que d'autres systèmes enzymatiques peuvent être affectés par le parasitisme hépatique. L'efficacité des enzymes de conjugaison demeure inchangée 4 ou 8 semaines après l'infestation par Fasciola hepatica, ces stades correspondant à la migration intraparenchymateuse des larves à travers le foie (4 semaines) où à l'apparition des formes adultes dans les canicules biliaires (8 semaines). En conclusion, la fasciolose se classe parmi de nombreuses atteintes hépatiques susceptibles de perturber l'enzymologie hépatique de biotransformation des médicaments. En ce sens, des animaux atteints de parasitisme pourraient se trouver sensibilisés à certains médicaments. Le problème des résidus toxiques risquerait de se poser alors, dans des conditions différentes de celles d'animaux indemnes de parasitisme.

Galtier P., Battaglia A., More J., Franc M., 1983. Impairement of drug metabolism by the liver in experimental fascioliasis in the rat. J. Pharm. Pharmacol., 35, 729-733.

Galtier P., Larrieu G., Le Bars J., 1984. Comparative incidence of oral ochratoxicosis and aflatoxicosis on the activity of drug metabolizing enzymes in rat liver. Toxicol. Let, 23, 341-347.

Weber W. W., Glowinski I. B., 1980. Acetylation, 169-167. In Jakoby W. B., Enzymatic basis of detoxication, vol. II, Acad. Press, New York. 